\title{
Supplement of : UK Global Ocean GO6 and G07: a traceable hierarchy of model resolutions
}

David Storkey et al

Correspondence to: David Storkey (dave.storkey@metoffice.gov.uk)

\section{FPP keys used in GO6 and GSI8.1}

These tables contain the NEMO and CICE preprocessing keys required to build GO6-GSI8.1.

\begin{tabular}{ll}
\hline keys for building NEMO & \\
\hline key_cice & couple to CICE sea ice model \\
key_dynspg_flt & filtered free surface solver \\
key_vvl & z $^{*}$ vertical coordinate \\
key_ldfslp & rotate tracer diffusion operators \\
key_traldf_c2d & geographically varying lateral tracer diffusion \\
key_dynldf_c2d & geographically varying lateral momentum diffusion \\
key_zdftke & TKE scheme for vertical mixing \\
key_zdftmx & include tidal mixing scheme \\
key_trabbl & include bottom boundary layer scheme \\
key_zdfddm & include double diffusive mixing parametrisation \\
key_mpp_mpi & use MPI library \\
key_mpp_rep & global sums bit compare for parallel model runs with different grid decompositions \\
key_nosignedzero & overwrite f95 behaviour of intrinsic sign function \\
key_iomput & use XIOS I/O library \\
key_xios2 & use version 2 of XIOS I/O library \\
key_nemocice_decomp & use identical parallel decomposition for NEMO and CICE \\
key_traldf_eiv & $1^{\circ}$ model only : include eddy-induced advection \\
\hline
\end{tabular}




\section{keys for building CICE}

\section{LINUX}

CICE_IN_NEMO, key_nemocice_decomp ncdf

coupled, key_iomput

gather_scatter_barrier

ORCA_GRID

NICECAT $=5$, NICELYR=4, NSNWLYR=1

TRAGE $=1$, TRPND $=1$

REPRODUCIBLE

NXGLOB=360, NYGLOB=330

$\mathrm{NXGLOB}=1440, \mathrm{NYGLOB}=1205$

$\mathrm{NXGLOB}=4320, \mathrm{NYGLOB}=3604$
Building CICE for the Linux environment

CICE is run within the NEMO model on the same processor decomposition

NetCDF format options available for input and output files

CICE coupled to NEMO and passing variables through NEMO

Use MPI barrier for safer gather and scatter communications use the ORCA family of grids

5 thickness categories, 4 ice layers, 1 snow layer

Using single ice age and melt-pond tracers

Ensures global sums bit compare for parallel model runs with different grid decompositions

$1^{\circ}$ model : size of grid in $\mathrm{x}$ and $\mathrm{y}$ directions

$1 / 4^{\circ}$ model : size of grid in $\mathrm{x}$ and $\mathrm{y}$ directions

$1 / 12^{\circ}$ model : size of grid in $\mathrm{x}$ and $\mathrm{y}$ directions

\section{NEMO namelists}

This section contains the scientifically relevant namelists for the NEMO ocean model for GO6. This file should be included

5 as the namelist_cfg file in the working directory and other namelists will be set to default values by the namelist_ref file. The full scientific definition is give for the $1 / 4^{\circ}$ model, but for the $1^{\circ}$ and $1 / 12^{\circ}$ models only those namelist parameters that are changed compared to the $1 / 4^{\circ}$ model.

\subsection{Namelists for $1 / 4^{\circ}$ model}

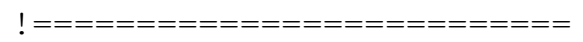

5 \&namrun

! $=======================$

nn_no $=0$

cn_exp $=$ 'go6 $620^{\prime}$

nn_it000 = 1

10 nn_itend $=1984$

nn_date0 = 19760101

$\mathrm{nn} \_$leapy $=0$

ln_rstart $=$.false.

nn_euler $=1$

15 nn_rstetl $=0$

cn_ocerst_in = 'restart' 
cn_ocerst_indir $=\prime . /$ '

cn_ocerst_out $=$ 'restart'

cn_ocerst_outdir $=$ '/home/d00/frsy/cylc-run/u-ah494-make-namelist/share/data/History_Data/

20 nn_istate $=1$

ln_rst_list $=$.false.

nn_stock = 10000

nn_stocklist $=10 * 0$

nn_write $=640$

5 ln_dimgnnn = .false.

ln_mskland $=$.true.

In_cfmeta $=$.true.

ln_clobber $=$.true.

nn_chunksz = 2097152

10 In_rstdate = .true.

/

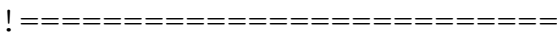

\&namcfg

$15 !==========================$

cp_cfg $=$ 'orca'

cp_cfz $=$ 'no zoom'

jp_cfg $=25$

jpidta $=1442$

20 jpjdta $=1207$

jpkdta $=75$

jpiglo = 1442

jpjglo = 1207

jpizoom = 1

25 jpjzoom = 1

jperio $=4$

In_use_jattr = .false.

/

30

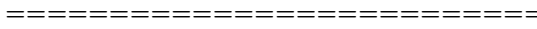

\&namzgr 


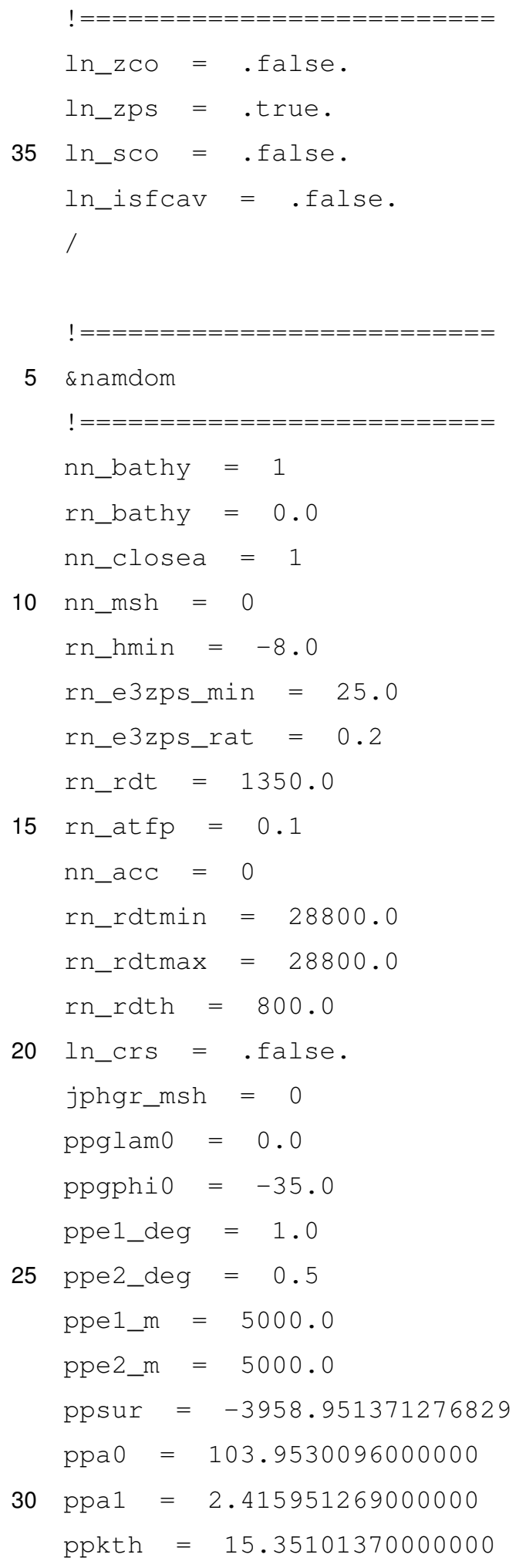


ppacr $=7.0$

ppdzmin $=999999.0$

pphmax = 999999.0

35 ldbletanh $=$.true.

ppa2 = 100.7609285000000

ppkth2 $=48.02989372000000$

ppacr $2=13.0$

/

5

! $======================$

\&namtsd

! $====================$

sn_tem = 'EN4_climatologies.nc',-1,'temperature',.true.,.true.,'yearly',' ', ', '

10 sn_sal = 'EN4_climatologies.nc',-1,'salinity',.true.,.true.,' 'yearly', ', ,' ',

cn_dir = '/projects/ocean/hadgem3/initial/ocean/eorcA025/'

ln_tsd_init $=$.true.

ln_tsd_tradmp $=$.false.

/

15

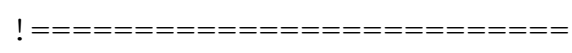

$\&$ namsbc

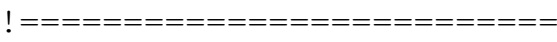

$\mathrm{nn} \_\mathrm{fsbc}=1$

20 In_ana $=$.false.

ln_flx $=$.false.

ln_blk_clio = ffalse.

ln_blk_core $=$.true.

ln_blk_mfs = .false.

25 In_cpl = .false.

ln_mixcpl = .false.

nn_components $=0$

ln_apr_dyn = .false.

nn_ice $=4$

30 nn_ice_embd $=1$

ln_dm $2 \mathrm{dc}=$.true. 


$$
\begin{aligned}
& \text { In_rnf }=\text {.true. } \\
& \text { nn_isf }=3 \\
& \text { In_ssr }=\text {.true. } \\
& 35 \text { nn_fwb }=0 \\
& \text { In_wave }=\text {.false. } \\
& \text { In_cdgw }=\text {.false. } \\
& \text { ln_sdw }=\text {.false. } \\
& \mathrm{nn} \_ \text {lsm }=0 \\
& 5 \text { nn_limflx }=-1 \\
& \text { / }
\end{aligned}
$$

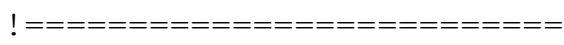

\&namsbc_core

10

sn_wndi = 'u10_core',6,'U_10_MOD', .true.,.false.,'yearly','weights_grid03_bicubic_eorca025 sn_wndj = 'v10_core', 6,' V_10_MOD', ttrue.,.false.,'yearly','weights_grid03_bicubic_eorca025 sn_qsr = 'qsw_core',24,'SWDN_MOD', ffalse.,.false.,'yearly','weights_grid03_bilinear_eorca0 sn_qlw = 'qlw_core',24,'LWDN_MOD', ffalse., false.,'yearly',' weights_grid03_bilinear_eorca0

15 sn_tair = 't10_core',6,'T_10_MOD', ttrue.,ffalse.,'yearly','weights_grid03_bilinear_eorca02 sn_humi = 'q10_core',6,'Q_10_MOD', .true.,.false.,'yearly','weights_grid03_bilinear_eorca02 sn_prec = 'precip_core',-1,'TPRECIP', .true.,.false.,'yearly', 'weights_grid03_bilinear_eorc sn_snow = 'snow_core',-1,' SNOW', true.,.false.,'yearly',' weights_grid03_bilinear_eorca025. sn_tdif = 'taudif_core',24,'TAUDIF', ttrue.,.false.,'yearly',' weights_grido3_bilinear_eorca

20 cn_dir = '/projects/ocean/hadgem3/forcing/ocean/CORE2/' ln_taudif $=$.false.

$r n \_z q t=10.0$

rn_zu $=10.0$

rn_pfac $=1.0$

25 rn_efac $=1.0$

rn_vfac $=1.0$

/

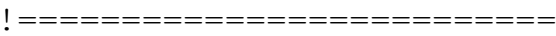

30 \&namtra_qsr

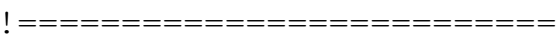




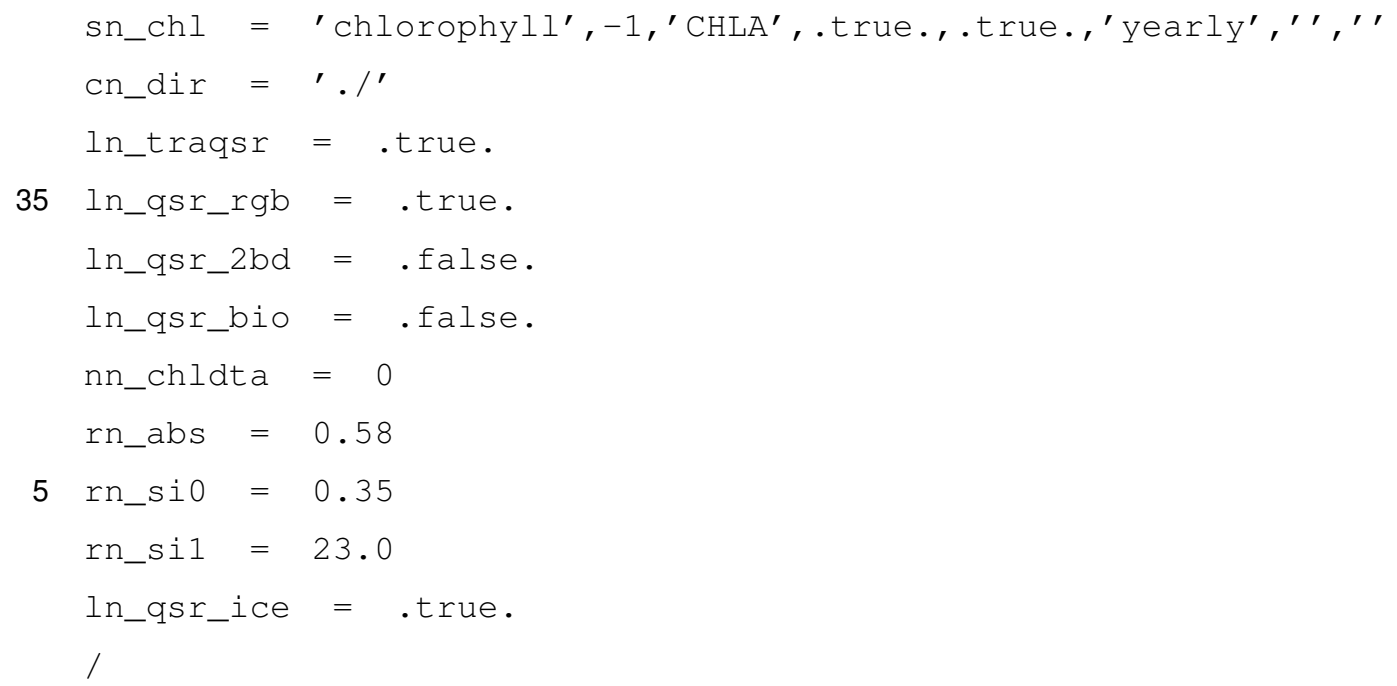

20 rn_hrnf $=10.0$

rn_avt_rnf $=2.0 e^{-3}$

rn_rfact $=1.0$

ln_rnf_depth $=$.false.

ln_rnf_tem = false.

25 ln_rnf_sal = .false.

ln_rnf_depth_ini $=$.false.

rn_rnf_max $=5.735 \mathrm{e}-4$

rn_dep_max $=150.0$

nn_rnf_depth_file $=0$ 


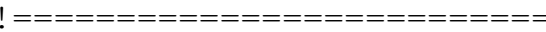

\&namsbc_isf

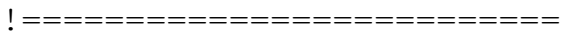

35 sn_qisf = 'rnfisf',-12,'sohflisf', .false.,.true.,'yearly', ', ',' '

sn_fwfisf = 'rnfisf',-12,'sowflisf',.false.,.true.,'yearly', ', ,' '

sn_rnfisf $=$ 'runoff_lm_nomask', $-1,{ }^{\prime}$ sofwfisf',.true.,.true.,'yearly', ' ', ' '

sn_depmax_isf = 'runoff_lm_nomask',-1,'sozisfmax',.false.,.true.,'yearly',' ',' '

sn_depmin_isf = 'runoff_lm_nomask',-1,'sozisfmin',.false.,.true.,'yearly',' ',' '

5 sn_Leff_isf = 'rnfisf',0,' Leff',.ffalse.,.true.,' yearly', ' ',' '

ln_divisf $=$.true.

rn_gammat $0=1.0 e-4$

rn_gammas $0=1.0 e-4$

$\mathrm{nn}$ _isfblk $=1$

10 rn_hisf_tbl $=30.0$

ln_conserve $=$.true.

$\mathrm{nn}$ _gammablk = 1

/

15

$\&$ namsbc_apr

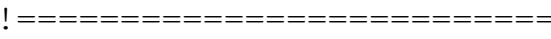

sn_apr = 'patm', -1, 'somslpre', .true.,.true.,'yearly',', ',' '

cn_dir $=$ '. /'

20 rn_pref $=101000.0$

ln_ref_apr $=$.false.

ln_apr_obc $=$.false.

/

25

\&namsbc_ssr

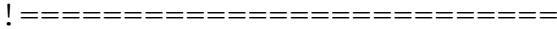

sn_sst = 'sst_data', 24,'sst',.ffalse.,.false.,'yearly', ', ',' ', ','

sn_sss = 'sss_1m.nc',-1,'vosaline',.true.,.true.,'yearly',' ', ', '

30 cn_dir $=$ '. /'

nn_sstr $=0$ 


$$
\begin{aligned}
& \text { nn_sssr }=2 \\
& \text { rn_dqdt }=-40.0 \\
& \text { rn_deds }=-33.333333
\end{aligned}
$$

35 In_sssr_bnd $=$.true.

rn_sssr_bnd $=4.0$

/

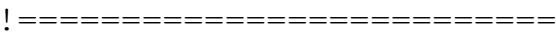

5 \&namsbc_alb

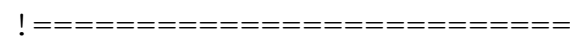

$n n \_i c e \_a l b=0$

rn_albice $=0.53$

rn_alphc $=0.65$

10 rn_alphd $=0.80$

rn_alphdi $=0.72$

rn_cloud $=0.06$

/

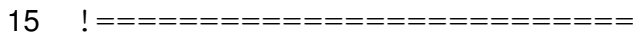

\&namberg

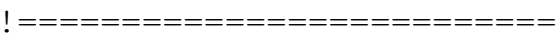

In_icebergs $=$.true.

In_bergdia $=$.true.

20 nn_verbose_level $=1$

nn_verbose_write $=15$

nn_sample_rate $=64$

rn_initial_mass $=8.8 \mathrm{e} 7,4.1 \mathrm{e} 8,3.3 \mathrm{e} 9,1.8 \mathrm{e} 10,3.8 \mathrm{e} 10,7.5 \mathrm{e} 10,1.2 \mathrm{e} 11,2.2 \mathrm{e} 11,3.9 \mathrm{e} 11,7.4 \mathrm{e} 11$

rn_distribution $=0.24,0.12,0.15,0.18,0.12,0.07,0.03,0.03,0.03,0.02$

25 rn_mass_scaling $=2000,200,50,20,10,5,2,1,1,1$

rn_initial_thickness $=40.0,67.0,133.0,175.0,6 * 250.0$

rn_rho_bergs $=850.0$

rn_Low_ratio $=1.5$

ln_operator_splitting $=$.true.

30 rn_bits_erosion_fraction $=0$

rn_sicn_shift $=0$ 
In_passive_mode $=$.false.

nn_test_icebergs $=-1$

rn_test_box $=108.0,116.0,-66.0,-58.0$

35 rn_speed_limit $=0.0$

sn_icb = 'calving',-1,' calvingmask', .true.,.true.,' yearly', ,' ,','

cn_dir $=\prime . / \prime$

rn_low_ratio $=1.5$

/

5

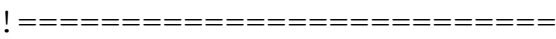

\&namlbc

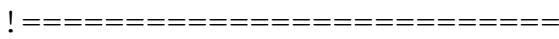

rn_shlat $=0.0$

10 In_vorlat $=$.false.

/

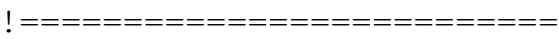

\&namcla

15 ! $=========================$

$\mathrm{nn}$ _cla $=0$

/

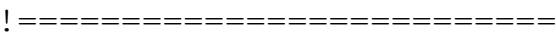

20 \&nambfr

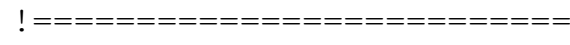

nn_bfr $=2$

rn_bfril $=4.0 e-4$

rn_bfri2 = $1.0 e-3$

25 rn_bfri2_max $=1.0 e-1$

rn_bfeb2 $=2.5 e-3$

rn_bfrzo $=3.0 e-3$

In_bfr2d $=$.true.

rn_bfrien $=50.0$

30 rn_tfril $=4.0 e-4$

rn_tfri2 $=2.5 e-3$ 


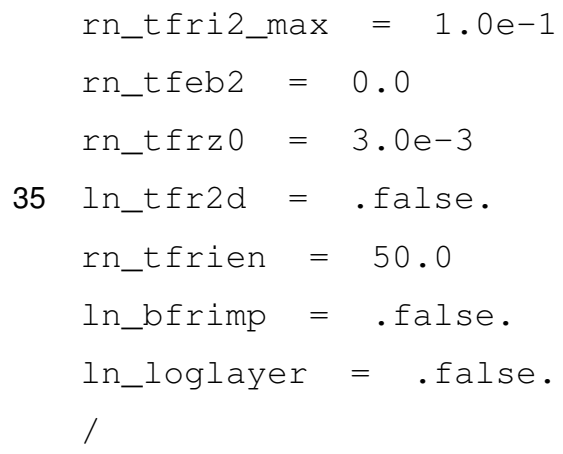

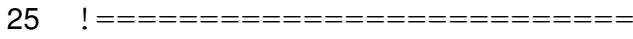
\&nameos

! $=\mathrm{e=}=\mathrm{=}=\mathrm{=}=\mathrm{=}=\mathrm{=}=\mathrm{=}=\mathrm{=}=\mathrm{=}=\mathrm{=}=\mathrm{=}=\mathrm{=}$

nn_eos $=0$

In_usect $=$.true.

30 rn_a0 $=1.6550 e-1$

rn_bo $=7.6554 \mathrm{e}-1$ 


$$
\begin{aligned}
\text { rn_lambdal }=5.9520 \mathrm{e}-2 \\
\text { rn_lambda2 }=7.4914 \mathrm{e}-4 \\
\text { rn_mu1 }=1.4970 \mathrm{e}-4 \\
35 \text { rn_mu2 }=1.1090 \mathrm{e}-5 \\
\text { rn_nu }=2.4341 \mathrm{e}-3 \\
\text { ln_usect }=\text {.false. }
\end{aligned}
$$

$5 \mathrm{l}=\mathrm{=}=\mathrm{=}=\mathrm{=}=\mathrm{=}=\mathrm{=}=\mathrm{=}=\mathrm{=}=\mathrm{=}=\mathrm{=}=\mathrm{=}=\mathrm{=}=\mathrm{=}$ \&namtra_adv

$!========================$

ln_traadv_cen2 = .false.

ln_traadv_tvd = .true.

10 In_traadv_muscl = .false.

In_traadv_muscl2 = .false.

ln_traadv_ubs = .false.

ln_traadv_qck = .false.

ln_traadv_msc_ups $=$.false.

15 In_traadv_tvd_zts = .false. /

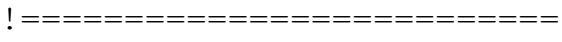
\&namtra_adv_mle

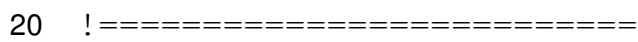
ln_mle $=$.false.

rn_ce $=0.06$

nn_mle $=1$

rn_lf $=5.0 e+3$

25 rn_time $=172800.0$

rn_lat $=20.0$

nn_mld_uv $=0$

$\mathrm{nn}$ _conv $=0$

rn_rho_c_mle $=0.01$

$30 \%$ 


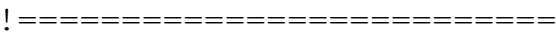

\&namtra_ldf

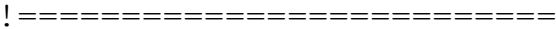

35 In_traldf_lap $=$.true.

ln_traldf_bilap $=$.false.

ln_traldf_level = .false.

ln_traldf_hor $=$.false.

ln_traldf_iso $=$.true.

5 ln_traldf_grif $=$.false.

ln_traldf_gdia $=$.false.

In_triad_iso $=$.false.

ln_botmix_grif $=$.false.

rn_aeiv_0 $=0.0$

10 rn_aht_o $=150.0$

rn_ahtb_0 $=0.0$

rn_slpmax $=0.01$

rn_chsmag $=1.0$

rn_smsh $=1.0$

15 rn_aht_m $=2000.0$

/

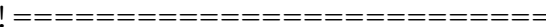
\&namtra_dmp

20 ! $========================$ ln_tradmp $=$.false.

$\mathrm{nn}$ _zdmp $=0$

cn_resto = 'resto.nc'

/

25

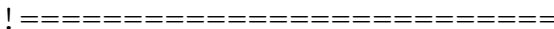

\&namdyn_adv

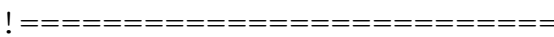

ln_dynadv_vec = .true.

30 nn_dynkeg = 1

ln_dynadv_cen2 = .false. 


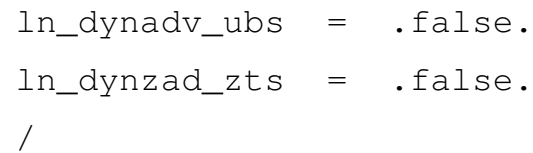

15

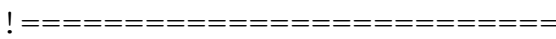

\&namdyn_vor

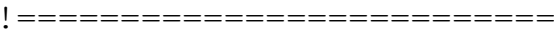

ln_dynvor_ene $=$.false.

20 In_dynvor_ens = .false.

ln_dynvor_mix = .false.

ln_dynvor_een $=$.false.

ln_dynvor_een_old = .true. /

25

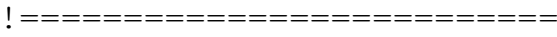
\&namdyn_hpg

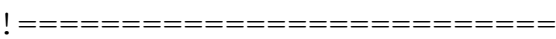
ln_hpg_zco $=$.false.

30 In_hpg_zps $=$.false.

ln_hpg_sco $=$.true. 
In_hpg_isf $=$.false.

In_hpg_djc $=$.false.

In_hpg_prj $=$.false.

35 In_dynhpg_imp $=$.true.

/

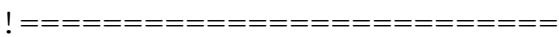

\&namdyn_ldf

5

ln_dynldf_lap $=$.false.

ln_dynldf_bilap $=$.true.

ln_dynldf_level $=$.false.

In_dynldf_hor $=$.true.

10 In_dynldf_iso $=$.false.

rn_ahm_0_lap $=40000.0$

rn_ahmb_o = 0.0

rn_ahm_0_blp $=-1.5 \mathrm{e} 11$

rn_cmsmag_1 $=3.0$

15 rn_cmsmag_2 $=3$

$r n \_c m s h=1.0$

rn_ahm_m_blp $=-1.0 e 12$

rn_ahm_m_lap $=40000.0$

I

20

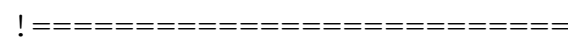

\&namzdf

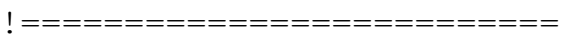

rn_avm0 $=1.2 e^{-4}$

25 rn_avt0 $=1.2 \mathrm{e}-5$

nn_avb = 0

nn_havtb = 1

In_zdfevd $=$.true.

nn_evdm $=1$

30 rn_avevd $=10.0$

ln_zdfnpc $=$.false. 


$$
\begin{aligned}
& \text { nn_npc }=1 \\
& \text { nn_npcp }=365 \\
& \text { ln_zdfexp }=. \text { false. } \\
& 35 \text { nn_zdfexp }=3 \\
& \text { / }
\end{aligned}
$$

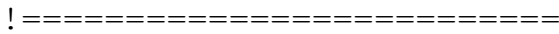
\&namzdf_tke

5

$$
\begin{aligned}
& \text { rn_ediff }=0.1 \\
& \text { rn_ediss }=0.7 \\
& \text { rn_ebb }=67.83 \\
& \text { rn_emin }=1.0 \mathrm{e}-6
\end{aligned}
$$

10 rn_emino $=1.0 e-4$

rn_bshear $=1.0 e-20$

$n n \_m x I=3$

$\mathrm{nn} \_\mathrm{pdl}=1$

ln_mxio = .true.

15 rn_mxlo $=0.04$

ln_lc $=$.true.

$r n \_l c=0.15$

nn_etau $=1$

rn_efr $=0.05$

20 nn_htau $=4$

/

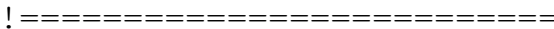
\&namzdf_ddm

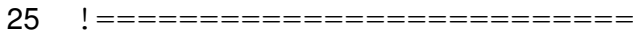
rn_avts $=1 \cdot 0 e^{-4}$

rn_hsber $=1.6$ /

$30 \mathrm{l}=\mathrm{=}=\mathrm{=}=\mathrm{=}=\mathrm{=}=\mathrm{=}=\mathrm{=}=\mathrm{=}=\mathrm{=}=\mathrm{=}=\mathrm{=}=\mathrm{=}=\mathrm{=}=\mathrm{=}$ $\&$ namzdf_tmx 


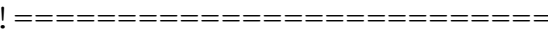

rn_htmx $=500.0$

$r n \_n 2 m i n=1.0 e-8$

35 rn_tfe $=0.333$

$r n \_m e=0.2$

In_tmx_itf $=$.true.

rn_tfe_itf $=1.0$

/

5

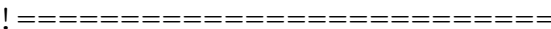

\&namzdf_mldzint

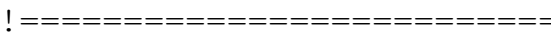

nn_mld_diag = 2

10 sn_mldl $=1,10.0,0.2,0.1$

$\mathrm{sn \_ mld2}=1,10.0,-0.2,0$

sn_mld3 $=0,0.0,0.0,0.0$

sn_mld4 $=0,0.0,0.0,0.0$

sn_mld5 $=0,0.0,0.0,0.0$

$15 /$

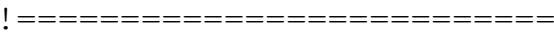
$\&$ nammpp

l $\mathrm{l}=\mathrm{=}=\mathrm{=}=\mathrm{=}=\mathrm{=}=\mathrm{=}=\mathrm{=}=\mathrm{=}=\mathrm{=}=\mathrm{=}=\mathrm{=}=\mathrm{=}=\mathrm{=}$

20 cn_mpi_send $=$ ' $I$ '

nn_buffer $=0$

ln_nnogather $=$.true.

jpni $=20$

jpnj $=24$

25 jpnij $=480$

/

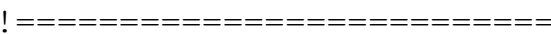

\&namct 1

30

ln_ctl $=$.false. 


$\begin{aligned} & \mathrm{nn} \text { _print }=0 \\ & \mathrm{nn} \text { _ictls }=0 \\ & \mathrm{nn} \text { _ictle }=0 \\ & 35 \mathrm{nn} \text { njctls }=0 \\ & \mathrm{nn} \text { _jctle }=0 \\ & \mathrm{nn} \text { _isplt }=1 \\ & \mathrm{nn} \text { _jsplt }=1 \\ & \mathrm{nn} \text { _bench }=0 \\ & 5 \mathrm{nn} \text { _timing }=1 \\ & /\end{aligned}$

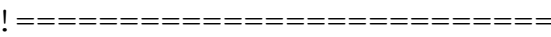

\&namnc4

10

nn_nchunks_i $=4$

nn_nchunks_j $=4$

nn_nchunks_k $=31$

ln_nc4zip $=$.true.

$15 /$

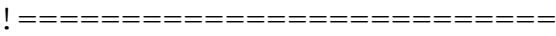

\&namt rd

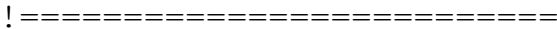

20 In_glo_trd $=$.false.

In_dyn_trd $=$.false.

ln_dyn_mxl $=$.false.

In_vor_trd $=$.false.

ln_kE_trd $=$.false.

25 In_PE_trd $=$.false.

In_tra_trd $=$.true.

ln_tra_mxl = ffalse.

nn_trd $=365$

ln_ke_trd $=$.false.

30 In_pe_trd $=$.false.

/ 
$\mathrm{l}=\mathrm{=}=\mathrm{=}=\mathrm{=}=\mathrm{=}=\mathrm{=}=\mathrm{=}=\mathrm{=}=\mathrm{=}=\mathrm{=}=\mathrm{=}=\mathrm{=}$

\&namptr

35

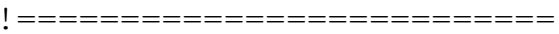

ln_diaptr $=$.false.

ln_subbas $=$.false.

/

5

\&namhsb

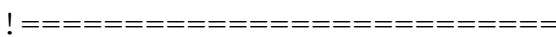

ln_diahsb = .false.

/

10

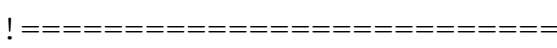

$\&$ namdct

! $====================$

$\mathrm{nn}$ _dct $=15$

15 nn_detwri $=15$

$\mathrm{nn}$ _secdebug $=112$

/

\subsection{Changed namelist parameters for $1^{\circ}$ model}

These are the namelist parameters that are changed compared to the list give for the $1 / 4^{\circ}$ model.

20

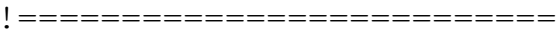

\&namcfg

$!===================$

cp_cfg = 'orca'

jp_cfg = 1

25 jpidta $=362$

jpjdta $=332$

jpkdta $=75$

jpiglo = 362

jpjglo = 332

30 


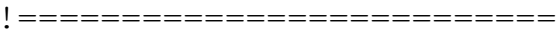

\&namdom

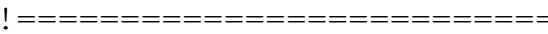

$r n \_r d t=2700.0$

/

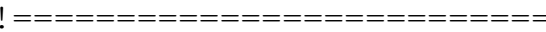

5 \&namberg

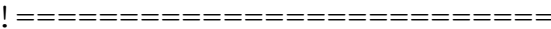

nn_sample_rate $=32$

/

10

\&namtra_ldf

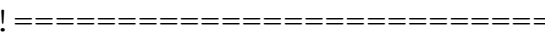

rn_aeiv_0 = 1000.0

rn_aht_o = 1000.0

$15 /$

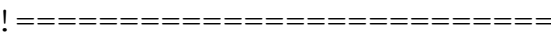
\&namdyn_ldf

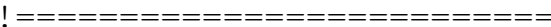

20 In_dynldf_lap = .true.

ln_dynldf_bilap = .false.

rn_ahm_0_lap = 20000.0

rn_ahm_0_blp $=0.0$

/

25

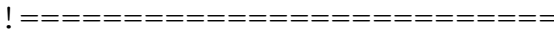
$\&$ nammpp

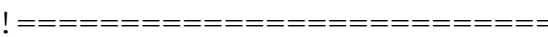
cn_mpi_send $=$ ' $I$ '

30 nn_buffer $=0$

ln_nnogather $=$.true. 
jpni $=22$
jpnj $=21$
jpnij $=462$
$/$

\subsection{Changed namelist parameters for $1 / 12^{\circ}$ model}

These are the namelist parameters that are changed compared to the list give for the $1 / 4^{\circ}$ model.

\begin{tabular}{|c|c|c|}
\hline \multicolumn{3}{|l|}{$\&$ namcfg } \\
\hline \multicolumn{3}{|c|}{ 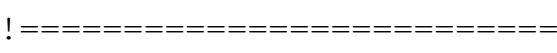 } \\
\hline cp_cfg & $=$ & 'orca' \\
\hline$j p \_c f g$ & $=$ & 12 \\
\hline jpidta & $=$ & 4322 \\
\hline jpjdta & $=$ & 3606 \\
\hline jpkdta & $=$ & 75 \\
\hline jpiglo & $=$ & 4322 \\
\hline jpjglo & $=$ & 3606 \\
\hline / & & \\
\hline
\end{tabular}

$15 !======================$ \&namdom

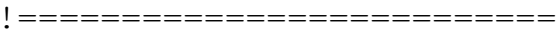

rn_rdt $=360.0$

/

20

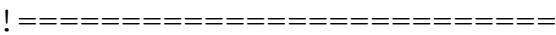
\&namberg

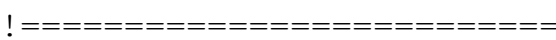
nn_verbose_write $=1200$

25 nn_sample_rate $=1200$ 1

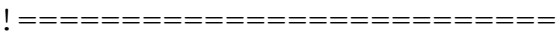
$\&$ namlbc

30 ! $=======================$ 


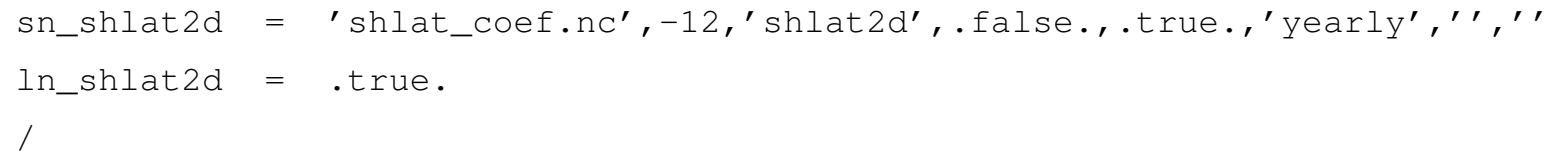

\section{CICE namelists}

This section contains the CICE namelists. The full namelists are given for the $1 / 4^{\circ}$ model and then for the $1^{\circ}$ and $1 / 12^{\circ}$ models 25 only those namelist parameters that are changed compared to the $1 / 4^{\circ}$ model.

\subsection{Namelists for $1 / 4^{\circ}$ model}

$\&$ setup_nml

days_per_year $=365$, 
dbug $=$. false. ,

30 diag_file='cice_standard_output' ,

diag_type='file',

diagfreq $=320$,

$d t=1350.0$,

dump_last=.true.,

dump freq $={ }^{\prime} \mathrm{m}{ }^{\prime}$,

dumpfreq_n $=1$,

5 hist_avg=.true.,

histfreq=' $\mathrm{m}^{\prime},{ }^{\prime} \mathrm{d}^{\prime},{ }^{\prime} \mathrm{x}^{\prime},{ }^{\prime} \mathrm{x}^{\prime},{ }^{\prime} \mathrm{x}^{\prime}$,

histfreq_n $=5 * 1$,

history_dir='./',

history_file='go6x2i.1m',

10 ice_ic='/projects/ocean/hadgem3/initial/seaice/eoRCA025//aoksv.01.12.2019.2038.multilayer.ex incond_dir=' . /',

incond_file='go6 2 i_ic',

istep $0=0$,

latpnt $(1)=90.0$,

15 latpnt $(2)=-65.0$,

lnetcdf $4=$. true. ,

lonpnt $(1)=0.0$,

lonpnt $(2)=-45.0$,

$n d t d=1$,

20 npt $=1984$,

pointer_file='/home/d00/frsy/cylc-run/u-ah494-make-namelist/share/data/History_Data/CICEhist print_global=.true.,

print_points=.true.,

restart $=$.true.,

25 restart_dir='/home/d00/frsy/cylc-run/u-ah494-make-namelist/share/data/History_Data/CICEhist/ restart_ext=.true.,

restart_file='go6x2i.restart',

restart_format $=^{\prime}$ nc',

runtype='initial',

30 use_leap_years=.false. ,

use_restart_time=.false., 
write_ic=.true.,

year_init $=1976$,

/

35 \&grid_nml

grid_file='/projects/ocean/hadgem3/grids/seaice/eORCA025/CICE_grid_orca025ext_nemo_dist.nc', grid_format $={ }^{\prime}$ nc',

grid_type='tripole',

kcatbound=1,

5 kmt_file='/projects/ocean/hadgem3/grids/seaice/eoRCA025/CICE_kmt_eorca025_v2.2_nemo_dist.nc' /

\&domain_nml

distribution_type $=^{\prime}$ nemo',

distribution_wght $={ }^{\prime}$ block',

10 ew_boundary_type=' cyclic',

maskhalo_bound=.false.,

maskhalo_dyn=.false.,

maskhalo_remap=.false.,

nprocs $=480$,

15 ns_boundary_type='tripoleT' ,

processor_shape $=$ ' square-pop' ,

/

\&tracer_nml

restart_aero=.false.,

20 restart_age $=$. false.,

restart_fy=.false.,

restart_lvl=.false.,

restart_pond_cesm=.false.,

restart_pond_lvl=.false.,

25 restart_pond_topo=.false.,

tr_aero=.false.,

tr_fy=.false.,

tr_iage=.true.,

tr_lvl=.false.,

30 tr_pond_cesm=.false. ,

tr_pond_lvl=.false., 
tr_pond_topo=.true.,

/

\&thermo_nml

35 a_rapid_mode $=0.5 e-3$,

aspect_rapid_mode $=1.0$,

conduct $={ }^{\prime}$ MU71',

dsdt_slow_mode $=-1.5 e-7$,

$\mathrm{kit} d=1$,

5 ktherm=1,

phi_c_slow_mode $=0.05$,

phi_i_mushy $=0.85$,

rac_rapid_mode $=10.0$,

saltmax $=9.6$,

$10 /$

\&dynamics_nml

advection=' remap',

$\mathrm{kdyn}=1$,

krdg_partic=1,

15 krdg_redist $=1$,

kstrength $=1$,

mu_rdg=3.0,

ndte $=120$,

revised_evp=.false.,

$20 /$

\&shortwave_nml

$\operatorname{ahmax}=0.5$,

albedo_type=' default',

albicei=0.36,

25 albicev=0.833,

albsnowi $=0.70$,

albsnowv $=0.98$,

$r_{\text {_ice }}=0.0$,

r_pnd $=0.0$,

30 r_snw $=1.5$,

rsnw_mlt $=1500.0$, 
shortwave=' default',

/

\&ponds_nml

35 dpscale=1.0e-3,

frzpnd=' cesm',

$\mathrm{hp} 1=0.01$,

hs $0=0.03$,

$\mathrm{hs} 1=0.03$,

5 pndaspect $=0.8$, rfracmax $=0.85$, rfracmin=0.15,

/

\&zbgc_nml

10 bgc_data_dir='unknown_bgc_data_dir', bgc_flux_type $={ }^{\prime}$ Jin2006', nit_data_type='default', phi_snow $=0.5$, restart_bgc=.false.,

15 restart_hbrine=.false., restore_bgc=.false.,

sil_data_type=' default', skl_bgc $=$.false., tr_bgc_am_sk=.false.,

20 tr_bgc_c_sk=. false., tr_bgc_chl_sk=.false. , tr_bgc_dms_sk=.false., tr_bgc_dmspd_sk=.false., tr_bgc_dmspp_sk=.false.,

25 tr_bgc_sil_sk=.false., tr_brine=.false.,

/

\&forcing_nml

atm_data_dir='unknown_atm_data_dir:' ,

30 atm_data_format $={ }^{\prime}$ nc',

atm_data_type='default', 
atmbndy=' default',

calc_strair=.true.,

calc_tsfc=.true.,

35 cap_fluxes=.true. ,

formdrag=.false.,

fyear_init=1997,

oceanmixed_file=' unknown_oceanmixed_file',

oceanmixed_ice=.false.,

5 ocn_data_dir='unknown_ocn_data_dir:' ,

ocn_data_format $={ }^{\prime}$ nc',

precip_units $=^{\prime} \mathrm{mks} \mathrm{s}^{\prime}$,

restore_ice $=$.false.,

restore_sst=.false.,

10 sss_data_type=' default',

sst_data_type=' default',

trestore $=0$,

update_ocn_f=.true.,

ustar_min $=5.0 e-4$,

15 ycycle=1,

/

\&icefields_nml

f_aice $={ }^{\prime} \mathrm{m}^{\prime}$,

f_aicen=' $\mathrm{m}^{\prime}$,

$20 \mathrm{f}$ _aisnap $={ }^{\prime} \mathrm{x}^{\prime}$,

f_albice $={ }^{\prime} x^{\prime}$,

f_albpnd $={ }^{\prime} x^{\prime}$,

f_albsni $=^{\prime} \mathrm{m}^{\prime}$,

f_albsno $={ }^{\prime} x^{\prime}$,

25 f_alidr $={ }^{\prime} x^{\prime}$,

f_alvdr $={ }^{\prime} \mathrm{x}^{\prime}$,

f_angle=.true.,

f_anglet $=$.true.,

f_bounds=.true. ,

$30 \mathrm{f}$ _congel $={ }^{\prime} \mathrm{m}$ ',

f_coszen ${ }^{\prime} x^{\prime}$, 
f_daidtd=' $\mathrm{m}^{\prime}$,

f_daidt $t={ }^{\prime} \mathrm{m}^{\prime}$,

f_divu=' m',

35 f_dsnow $={ }^{\prime} x^{\prime}$,

f_dvidt $d={ }^{\prime} \mathrm{m}^{\prime}$,

f_dvidt $t={ }^{\prime} \mathrm{m}^{\prime}$,

f_dxt=.false.,

f_dxu=.false.,

5 f_dyt=.false.,

f_dyu=.false.,

f_evap $=^{\prime} x^{\prime}$,

f_evap_ai=' $\mathrm{m}^{\prime}$,

f_fcondtop_ai $=^{\prime} x^{\prime}$,

10 f_fcondtopn_ai $=^{\prime} x^{\prime}$,

f_fhocn $=^{\prime} x^{\prime}$,

f_fhocn_ai=' m',

f_flat $=^{\prime} x^{\prime}$,

f_flat_ai='m',

15 f_flatn_ai=' $x^{\prime}$,

f_flwdn=' $\mathrm{m}^{\prime}$,

f_flwup $={ }^{\prime} x^{\prime}$,

f_flwup_ai $={ }^{\prime} \mathrm{m}^{\prime}$,

f_fmeltt_ai=' $x^{\prime}$,

20 f_fmelttn_ai=' $x^{\prime}$,

f_frazil='m',

f_fresh $=^{\prime} x^{\prime}$,

f_fresh_ai=' $\mathrm{m}^{\prime}$,

f_frz_onset $=^{\prime} \mathrm{m}^{\prime}$,

25 f_frzmlt $={ }^{\prime} \mathrm{m}^{\prime}$,

f_fsalt $={ }^{\prime} x^{\prime}$,

f_fsalt_ai='m',

f_fsens $=^{\prime} x^{\prime}$,

f_fsens_ai $=^{\prime} \mathrm{m}^{\prime}$,

30 f_fsurf_ai $=^{\prime} x^{\prime}$,

f_fsurfn_ai $=^{\prime} x^{\prime}$, 
f_fswabs $={ }^{\prime} x^{\prime}$,

f_fswabs_ai $={ }^{\prime} \mathrm{m}^{\prime}$,

f_fswdn $=^{\prime} \mathrm{m}^{\prime}$,

35 f_fswfac $={ }^{\prime} m^{\prime}$,

f_fswthru=' $x^{\prime}$,

f_fswthru_ai $=^{\prime} \mathrm{m}^{\prime}$,

$f_{f} \mathrm{f}_{\mathrm{y}}=^{\prime} \mathrm{x}^{\prime}$,

f_hi='m',

5 f_hisnap $={ }^{\prime} x^{\prime}$,

$\mathrm{f}_{\text {_h }} \mathrm{s}={ }^{\prime} \mathrm{m} \mathrm{\prime}^{\prime}$,

f_hte=.false.,

f_htn=.false.,

f_iage $={ }^{\prime} x^{\prime}$,

10 f_icepresent $={ }^{\prime} \mathrm{m}^{\prime}$,

f_meltb $={ }^{\prime} \mathrm{m}^{\prime}$,

f_meltl $={ }^{\prime} \mathrm{m}^{\prime}$,

f_melts $={ }^{\prime} \mathrm{m}^{\prime}$,

f_meltt $={ }^{\prime} \mathrm{m}^{\prime}$,

15 f_mlt_onset $=^{\prime} \mathrm{m}^{\prime}$,

f_ncat $=$.true.,

f_qref $={ }^{\prime} x^{\prime}$,

f_rain $={ }^{\prime} x^{\prime}$,

f_rain_ai $=^{\prime} \mathrm{m}^{\prime}$,

20 f_shear ${ }^{\prime} \mathrm{m}^{\prime}$,

f_sigl=' $\mathrm{m}^{\prime}$,

f_sig $2={ }^{\prime} \mathrm{m}^{\prime}$,

f_sinz $=^{\prime} \mathrm{m}^{\prime}$,

f_snoice $={ }^{\prime} \mathrm{m}^{\prime}$,

25 f_snow $=^{\prime} x^{\prime}$,

f_snow_ai $=^{\prime} \mathrm{m}^{\prime}$,

f_s $S{ }^{\prime}{ }^{\prime} \mathrm{m}^{\prime}$,

f_sst $=^{\prime} \mathrm{m}^{\prime}$,

f_strair $\mathrm{x}={ }^{\prime} \mathrm{m}^{\prime}$,

30 f_strairy $=^{\prime} \mathrm{m}^{\prime}$,

f_strcor $x={ }^{\prime} x^{\prime}$, 
f_strcory $={ }^{\prime} x^{\prime}$,

f_strength $=^{\prime} \mathrm{m}^{\prime}$,

f_strint $x={ }^{\prime} x^{\prime}$,

35 f_strinty $=^{\prime} x^{\prime}$,

f_strocn $x={ }^{\prime} x^{\prime}$,

f_strocny $=^{\prime} x^{\prime}$,

f_strtlt $x={ }^{\prime} x^{\prime}$,

f_strtlty $=^{\prime} x^{\prime}$,

5 f_tair $={ }^{\prime} \mathrm{m}^{\prime}$,

f_tarea=.true. ,

f_tin $z={ }^{\prime} \mathrm{m}^{\prime}$,

f_tmask=.true.,

f_tref $=^{\prime} x^{\prime}$,

10 f_trsig $=^{\prime} \mathrm{m}^{\prime}$,

f_tsf $\mathrm{C}^{\prime} \mathrm{m}^{\prime}$,

f_tsn $z={ }^{\prime} \mathrm{m}^{\prime}$,

f_uarea=.true.,

f_uocn=' $\mathrm{m}^{\prime}$,

15 f_uvel=' $\mathrm{m}^{\prime}$,

f_vgrdb=.false.,

f_vgrdi=.false.,

f_vgrds=.false.,

f_vicen $=^{\prime} \mathrm{m}^{\prime}$,

$20 \mathrm{f} \_\mathrm{vocn}=\mathrm{\prime}^{\prime} \mathrm{m}^{\prime}$,

f_vvel=' $\mathrm{m}^{\prime}$,

/

\&icefields_mechred_nml

f_alvl=' $x^{\prime}$,

25 f_aparticn $=^{\prime} x^{\prime}$,

f_araft $n={ }^{\prime} x^{\prime}$,

f_ardg $={ }^{\prime} x^{\prime}$,

f_ardgn $=^{\prime} x^{\prime}$,

f_aredist $n=^{\prime} x^{\prime}$,

30 f_dardg1 $d t={ }^{\prime} \mathrm{m}^{\prime}$,

f_dardg1ndt $={ }^{\prime} \mathrm{x}^{\prime}$, 
f_dardg $2 d t={ }^{\prime} \mathrm{m}^{\prime}$ ，

f_dardg2 $\mathrm{ndt}={ }^{\prime} \mathrm{x}^{\prime}$,

f_dvirdgdt $={ }^{\prime} \mathrm{m}^{\prime}$,

35 f_dvirdgndt $={ }^{\prime} x^{\prime}$,

$f \_k r d g n=x^{\prime}$,

f_opening $=^{\prime} \mathrm{m}^{\prime}$,

f_vlvl $=^{\prime} x^{\prime}$,

f_vraftn $=^{\prime} x^{\prime}$,

5 f_vrdg $={ }^{\prime} x^{\prime}$,

f_vrdgn $=^{\prime} \mathrm{x}^{\prime}$,

f_vredist $=^{\prime} x^{\prime}$,

/

\&icefields_pond_nml

10 f_apeff $f{ }^{\prime} \mathrm{m}^{\prime}$,

f_apeff_ai=' m',

f_apef $\mathrm{f} n=^{\prime} \mathrm{m}^{\prime}$,

f_apond $={ }^{\prime} \mathrm{m}^{\prime}$,

f_apond_ai $={ }^{\prime} \mathrm{m}^{\prime}$,

15 f_apondn $={ }^{\prime} \mathrm{m}^{\prime}$,

f_hpond $=^{\prime} \mathrm{m}^{\prime}$,

f_hpond_ai $={ }^{\prime} \mathrm{m}^{\prime}$,

f_hpondn=' m' ,

f_ipond $=^{\prime} \mathrm{m}^{\prime}$,

20 f_ipond_ai=' $\mathrm{m}^{\prime}$,

/

\subsection{Changed namelist parameters for $1^{\circ}$ model}

These are the namelist parameters that are changed compared to the list give for the $1 / 4^{\circ}$ model.

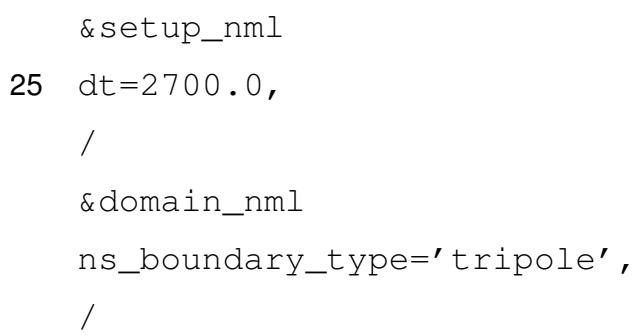




\section{3.3 Changed namelist parameters for $1 / 12^{\circ}$ model}

These are the namelist parameters that are changed compared to the list give for the $1 / 4^{\circ}$ model.

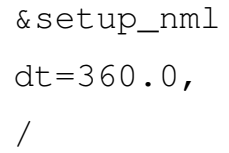

